\title{
Dopplerfluxometria de Ducto Venoso - Identificação não Invasiva da Acidemia em Fetos Prematuros Centralizados
}

\author{
Ductus Venosus Velocimetry - Noninvasive Identification of Fetal Acidemia in \\ Preterm Fetuses with Brain Sparing Reflex
}

Renato Augusto Moreira de Sá, Hermógenes Chaves Netto, Laudelino Marques Lopes, Paulo Roberto Nassar de Carvalho, Ynesmara Coelho Cosmo

\section{RESUMO}

Objetivo: estabelecer o ponto de corte a partir do qual seja possivel identificar fetos prematuros com centralização do fluxo sangüineo que apresentem gasometria anormal.

Métodos: foi realizado estudo observacional transversal, cuja população consistia de 60 gestantes com fetos centralizados (relação umbilico-cerebral maior que 1), com idade gestacional entre 25 e 33 semanas. O ducto venoso foi identificado com auxílio da dopplerfluxometria colorida e obtida a relação $S / A$ a partir do sonograma (relação entre a velocidade de pico da sístole ventricular e a velocidade de pico da sístole atrial). Imediatamente após a cesariana foi colhida amostra de sangue da veia umbilical para gasometria. Os conceptos foram classificados de acordo com a análise gasométrica e considerados anormais quando $\mathrm{pH}<7,20$ e $\mathrm{BE}<-6 \mathrm{mmol} / \mathrm{L}$. Após o cálculo da sensibilidade e especificidade para cada ponto de corte foi construída a curva ROC (receiver operator characteristic).

Resultados: foram selecionadas 60 gestantes no periodo de janeiro de 1998 a janeiro de 2003. No momento do estudo a idade gestacional variou entre 25 e 33 semanas, média de 29,7 semanas ( $\pm 1,8$ semanas). Todos os fetos apresentavam-se centralizados; dentre eles 14 apresentavam gasometria anormal ao nascimento e 46 apresentavam gasometria normal. A prevalência de resultados de fetos com gasometria anormal no material estudado foi de 23,33\%. Observou-se associação significativa entre o resultado anormal do ducto venoso $e$ gasometria anormal ao nascimento $\left(\chi^{2}=784,44, p<0,00001\right)$. O ponto de corte da relação S/ A (onde a curva ROC "muda de tendência") foi 3,4.

Conclusão: a análise da relação $S / A$ do ducto venoso se mostra adequada para a predição não invasiva de gasometria anormal em fetos prematuros, centralizados, quando os valores são superiores a 3,4.

PALAVRAS-CHAVE: Dopplervelocimetria. Gasometria. Circulação fetoplacentária. Dopplerfluxometria.

\section{Introdução}

A evolução tecnológica observada em décadas recentes, possibilitando o desenvolvimento de métodos que facilitam a observação e o estudo do feto, tem permitido extraordinário avanço no en-

Centro Pré-Natal de Diagnóstico e Tratamento Laranjeiras Clinica Perinatal, Rio de Janeiro - RJ Correspondência:

Renato Augusto Moreira de Sá

Rua das Laranjeiras 445, sub-solo - Laranjeiras

22240-002 - Rio de Janeiro - RJ tendimento da fisiologia e da fisiopatologia do paciente intra-uterino. Esta evolução na propedêutica fetal, além de tornar possivel melhor conhecimento da doença, fornece subsídios para que o tratamento do paciente ainda não nascido possa ser instituído. As gestações com evolução desfavorável para o concepto chamam-se genericamente gestações de alto risco e se associam, no mais das vezes, ao sofrimento fetal crônico. Vários fatores complicam a gestação, determinando alterações na placenta, pelo geral secundárias ao comprometimento da vascularização uterina, 
com sérias repercussões no sistema de trocas materno-fetais, configurando a insuficiência placentária. O concepto agredido pelas trocas placentárias deficientes pode morrer antes, no decorrer ou depois do parto. A interrupção da prenhez por vezes favorece o produto, livre agora do ambiente intra-uterino inóspito ${ }^{1}$.

A repercussão no feto das agressões por ele sofridas, extrinsecas ou intrinsecas, depende, em essência, da época, da intensidade e do nivel celular em que estas agressões ocorreram. Desde há muito é sabido que quanto mais precoce for a ofensa, mais grave serão suas conseqüências para o concepto, com aumento da mortalidade, da morbidade e do impacto econômico ${ }^{2}$.

A probabilidade de óbito neonatal é extremamente elevada quando o parto ocorre até a $25^{\mathrm{a}}$ semana (acima de 80\%), e apresenta redução significativa a cada semana até a $33^{\mathrm{a}}$ semana de gestação. Quando se analisa a morbidade, as maiores taxas ocorrem em conceptos nascidos com peso entre 500 e $750 \mathrm{~g}$, caindo progressivamente a cada intervalo de $250 \mathrm{~g}$ de ganho ponderal ${ }^{2}$.

Aproximadamente $7 \%$ dos nascimentos nos Estados Unidos correspondem a conceptos com menos de $2.500 \mathrm{~g}$ e estes consomem mais de um terço dos recursos destinados à saúde para o primeiro ano de vida ${ }^{3}$. Detectar as condições intrauterinas normais e as modificações encontradas em suas eventuais alterações patológicas tem sido a preocupação constante dos pesquisadores. A natural limitação imposta à investigação em humanos e a relativa inacessibilidade do feto constituem os principais obstáculos a estas pretensões.

Até recentemente a monitorização da freqüência cardiaca fetal constituía a única possibilidade de rastreamento do feto asfixiado. Os métodos biofísicos ganharam relevância com o desenvolvimento da tecnologia eletrônica ao final dos anos 60. Dos diversos métodos propedêuticos investigados com o objetivo de avaliar a saúde do concepto, nenhum foi completamente bem sucedido na identificação do sofrimento fetal crônico ${ }^{4}$. Este se caracteriza por modificações bioquímicas do meio interno, ocasionadas pela redução dos intercâmbios feto-maternos e que suscitam ao feto reações compensatórias ${ }^{2}$. Entretanto, a dopplerfluxometria da circulação fetal pode fornecer informações importantes sobre o bem estar. $\mathrm{O}$ único recurso de que o concepto dispõe para se adaptar a estas condições é a reorganização da circulação, alterando individualmente a pós-carga dos ventrículos ${ }^{4}$. As alterações do fluxo sangüíneo na insuficiência placentária estão relacionadas à diminuição na resistência vascular da circulação cerebral (redução da pós-carga do ventrículo esquerdo) e aumento da resistência na circulação periférica (aumento da pós-carga do ventrículo direito), fenômeno descrito há décadas e conhecido em nosso meio como centralização ${ }^{4}$.

$\mathrm{O}$ crescimento intra-uterino restrito decorrente do suprimento diminuído de nutrientes representa adaptação favorável ao feto ${ }^{5}$. A diminuição do seu tamanho, o desvio preferencial da circulação para órgãos nobres (centralização), a aceleração da maturidade pulmonar e a policitemia representam estratégia inicial de adaptação que só se torna patológica quando a privação é extre$\mathrm{ma}^{5}$.

O risco da prematuridade deve ser comparado ao da acidemia grave, ambas apresentando elevados índices de mortalidade perinatal e morbidade em longo prazo. O estudo dos vasos venosos indica a descompensação fetal por apresentar a melhor correlação com o estado ácido básico fetal. Em outras palavras, a acidemia pode ser detectada pela dopplerfluxometria venosa ${ }^{6}$. Além disso, as condutas neonatais baseiam-se na gasometria do cordão obtida ao nascimento.

Por meio da ultra-sonografia, visualizamos o ducto venoso no plano sagital em continuação direta com a veia umbilical na direção da veia cava inferior $^{7}$, seguindo de maneira inclinada para posterior em direção à porção esquerda da veia cava, que se dilata na saída do ducto venoso ${ }^{8}$. Neste nível também se anastomosam com a veia cava as veias hepáticas esquerda e média9.

O sonograma do ducto venoso se apresenta com dois "picos" de onda: o primeiro corresponde à sístole ventricular, associado ao relaxamento atrial (S), e o segundo à diástole (D), período de enchimento passivo que é seguido por um nadir da onda, representando a fase de enchimento ventricular ativo (A) ou contração atrial ${ }^{9}$.

A velocidade máxima sistólica no ducto venoso é, em média, de 40 a $80 \mathrm{~cm} / \mathrm{s}^{7}$. Os picos de velocidade sistólica e diastólica aumentam com a idade gestacional, mas a sua relação se mantém constante na gravidez, como também a relação $\mathrm{S} /$ $\mathrm{A}$, que corresponde à razão entre a velocidade de pico da sístole ventricular (S) e a velocidade de pico na contração atrial (A $)^{9,10}$. As ondas de dopplerfluxometria do ducto venoso podem ser registradas precocemente entre a $9^{\mathrm{a}}$ e a $10^{\mathrm{a}}$ semana de gestação ${ }^{11}$. Os componentes sistólico e diastólico se tornam visíveis a partir da $11^{\mathrm{a}}$ semana. Entre a $12^{\mathrm{a}}$ e a $15^{\mathrm{a}}$ semana de gestação as curvas são identificadas em $89 \%$ das mulheres examinadas ${ }^{12}$.

A velocidade de fluxo no ducto venoso é aproximadamente três vezes maior do que a velocidade de fluxo na veia umbilical ou na cava inferior. 
Esta velocidade está relacionada ao gradiente de pressão entre a veia umbilical e o sistema venoso central, estimado entre 0 e $3 \mathrm{mmHg}^{13}$. Gradientes de pressão superiores a $22 \mathrm{mmHg}$ são observados durante o movimento de respiração fetal. $\mathrm{O}$ forame oval está fechado durante a contração atrial e a este tempo o fluxo no ducto venoso vai refletir o gradiente entre o átrio direito e o ventrículo direito ${ }^{14}$. Desta forma, alterações na hemodinâmica fetal manifestadas por aumento da pressão venosa central, associada à falência cardíaca, podem ser precedidas por alterações na velocidade de fluxo do ducto venoso, característica que pode ser usada para seu diagnóstico ${ }^{14}$.

Rizzo et al. ${ }^{15}$ investigaram 97 fetos pequenos para a idade gestacional (PIG) e compararam com 164 adequados para a idade gestacional, tendo observado aumento significativo na velocidade de pico do ducto venoso e da relação S/A nos PIG. Observaram, ainda, pior prognóstico perinatal para aqueles cuja relação $\mathrm{S} / \mathrm{A}$ estava acima do percentil 95 para o esperado para a idade gestacional.

Em estudo recente observamos associação significativa entre o fluxo anormal no ducto venoso e gasometria anormal ao nascimento. Quando analisamos este parâmetro (fluxo anormal no ducto venoso) para predição da gasometria anormal ao nascimento, a sensibilidade foi de $66 \%$, a especificidade de $94 \%$, o valor preditivo positivo de $83 \%$ e o valor preditivo negativo de $86 \%$. Neste estudo consideraram-se como ducto venoso anormal aqueles em que a relação S/A era maior do que o percentil 95 para a idade gestacional ${ }^{16}$. Entretanto, faz-se necessário estabelecer um ponto de corte da relação S/A a partir do qual seja possivel identificar o concepto com gasometria anormal. Em outras palavras, é importante avaliar o contrabalanço entre a sensibilidade e a especificidade deste método diagnóstico, para decidir o melhor ponto de corte na identificação dos fetos comprometidos.

O objetivo do estudo é, utilizando-se da dopplerfluxometria do ducto venoso, estabelecer o ponto de corte a partir do qual seja possivel identificar fetos prematuros com centralização do fluxo sangüineo que apresentam gasometria anormal.

\section{Pacientes e Métodos}

O estudo realizado foi do tipo observacional transversal para avaliação de teste de diagnóstico. Os dados foram colhidos prospectivamente e a amostragem foi feita de forma aleatória.
Todas as pacientes participantes do estudo foram informadas sobre os objetivos, segurança e os tipos de exames propostos para a pesquisa. $\mathrm{O}$ consentimento pós-informação foi obtido sem formular-se por escrito, de acordo com o artigo $13^{\circ}$, capítulo II da resolução número $01 / 88$ do Conselho Nacional de Saúde (CNS), por se tratar de pesquisa com risco mínimo (artigo $7^{\circ}$, parágrafo II, capítulo I da resolução $01 / 88$ do CNS).

Foram considerados como critérios de inclusão: a idade gestacional correta - todas as gestantes selecionadas apresentavam idade gestacional calculada em semanas completas pela data da última menstruação (DUM) - pelo critério adotado pela Organização Mundial de Saúde ${ }^{17}$ e referendado pela FIGO em $1976{ }^{17}$. A idade gestacional era confirmada com exames ultra-sonográficos de primeiro e segundo trimestre [comprimento cabeçanádega $(\mathrm{CCN})$, diâmetro biparietal (DBP) e comprimento do fêmur $(\mathrm{CF})$ ]. Todas as gestantes foram submetidas a cesariana sob anestesia peridural na ausência de trabalho de parto, tendo como indicação básica para o ato cirúrgico a préeclâmpsia ou a centralização fetal (não houve influência do resultado da dopplerfluxometria do ducto venoso na indicação da cesariana. Por não se tratar de exame de rotina, os médicos que assistiam às pacientes não eram informados do resultado da dopplerfluxometria do ducto venoso). Foram considerados como critérios de exclusão: a presença de anormalidades anatômicas identificadas à ultra-sonografia, a gestação múltipla e a colheita inadequada da amostra de sangue da veia umbilical para análise gasométrica após o nascimento.

Inicialmente realizou-se o perfil hemodinâmico fetal (dopplerfluxometria arterial, usando como referência a artéria cerebral média e a artéria umbilical) em todas as pacientes. A relação umbílico/cerebral (U/C) fetal foi definida por meio da razão entre o fluxo da artéria umbilical e da artéria cerebral média. A centralização fetal foi caracterizada quando a razão entre o índice de pulsatilidade (IP) da artéria umbilical (U) e da artéria cerebral média $(\mathrm{C})$ era maior ou igual a 1 $(\mathrm{U} / \mathrm{C} \geq 1)$. A seguir foi realizada a dopplerfluxometria do ducto venoso.

Os exames de dopplerfluxometria foram realizados utilizando-se transdutor convexo de 3,75 $\mathrm{MHz}$. Todas as medidas de dopplerfluxometria foram feitas na ausência de contrações uterinas e em período de absoluto repouso fetal, aí se incluindo os movimentos respiratórios. Foram determinados os pontos A (pico da sístole) e B (final da diástole), obtendo-se automaticamente as relações sístole/diástole (A/B), índice de resistência (RI) e IP, para as artérias umbilical e cerebral média. 
Para obtenção do sonograma da artéria cerebral média pela via transabdominal, realizavase secção horizontal do cérebro, incluindo o tálamo e o cavum do septo pelúcido (plano de corte habitual para a medida do diâmetro biparietal). Movendo-se o transdutor caudalmente em plano paralelo ao anterior em direção à base do crânio, foram identificadas as fossas anterior, média e posterior. As artérias cerebrais eram vistas com auxílio da dopplerfluxometria colorida como duas estruturas pulsando em estreita proximidade das asas maiores do esfenóide. Acionamos o recurso do Doppler pulsátil para obtenção das ondas de velocidade e realizamos as medições.

O sonograma da artéria umbilical foi obtido colocando-se o transdutor na parede abdominal materna e localizando-se com auxílio da dopplerfluxometria colorida os três segmentos do cordão umbilical: proximal (próximo à inserção na parede abdominal fetal), alça livre e distal (próximo à inserção placentária). Após acionarmos o Doppler pulsátil, colocamos a janela de modo que a veia umbilical fosse visualizada no mesmo corte, obtivemos as ondas de velocidade para cada segmento e realizamos as medições. Caso houvesse diferença do IP nos três segmentos, considerávamos a média destes para a relação U/C.

O ducto venoso foi identificado usando a imagem de ultra-sonografia em duas dimensões e dopplerfluxometria colorida. A primeira etapa foi a localização do sinus portal e da inserção do cordão umbilical na parede abdominal, visualizados em corte de secção transversal do abdome fetal ou por vezes médio-sagital. Sem perder esta visão, o transdutor era movido até que a origem do ducto venoso no sinus portal fosse identificada. A janela do Doppler pulsátil era então posicionada na origem do vaso (istmo) e por vezes em sua porção distal $^{18}$ e obtidas as ondas de velocidade. Foram medidas a velocidade de pico durante a sístole ventricular (S) e a velocidade correspondente à contração atrial (A) e calculada a relação $\mathrm{S} / \mathrm{A}^{19}$.

O tempo decorrido entre o diagnóstico de centralização fetal e o nascimento (cesariana sob anestesia peridural) foi de, no máximo, cinco horas. Para a colheita da amostra do sangue do cordão foram utilizadas seringas plásticas de $3 \mathrm{~mL}$ da marca Becton Dickinson (B-D) e agulha descartável da mesma marca (30 x $7-22 \mathrm{G})$. Foi utilizada heparina sódica em ampolas de $0,25 \mathrm{~mL}$ em solução aquosa. A heparina foi aspirada para a seringa e homogeneamente distribuída pelas paredes da mesma. A seguir esvaziava-se a seringa com a agulha voltada para cima, para expelir o ar remanescente (mediante esta técnica aproximadamente $0,2 \mathrm{~mL}$ da heparina permanecem na seringa e na agulha).
Imediatamente após o parto um segmento de cordão de aproximadamente 20 a $30 \mathrm{~cm}$ de comprimento era isolado e clampeado com duas pinças. A amostra de sangue $(2 \mathrm{~mL})$ era então colhida através da punção da veia umbilical com a seringa previamente preparada, sendo ocluída a seguir. As amostras eram enviadas para análise gasométrica em 10 minutos, em média, devidamente acondicionada em isopor com gelo. A análise do sangue foi feita no aparelho Radiomiter ABL-5 .

$\mathrm{O}$ resultado do $\mathrm{pH}$ foi analisado segundo os seguintes parâmetros: a acidemia foi definida quando o $\mathrm{pH}$ do sangue da veia umbilical era inferior a 7,20; a acidemia metabólica quando base excess $(\mathrm{BE})<-6 \mathrm{mmol} / 1$ e $\mathrm{pCO}_{2} \leq 50 \mathrm{mmHg}$. Por outro lado, consideramos acidemia respiratória quando $\mathrm{BE} \geq-$ $6 \mathrm{mmol} / \mathrm{L} \mathrm{e} \mathrm{pCO}_{2}>50 \mathrm{mmHg}$, e acidemia mista quando $\mathrm{BE}<-6 \mathrm{mmol} / \mathrm{L} \mathrm{e} \mathrm{pCO}_{2}>50 \mathrm{mmHg}$. Para efeito de análise estatística foram considerados como gasometria anormal aquelas que apresentavam acidemia metabólica ou mista, uma vez que estão associadas a aumento significativo de complicações neonatais, ao passo que a acidemia respiratória não apresenta a mesma associação ${ }^{20}$.

Foram considerados como PIG aqueles fetos que apresentavam ao nascimento peso inferior ao percentil 10 do esperado para a idade gestacional.

Inicialmente calculamos o tamanho amostral com auxílio do programa computacional Epi-Info versão 5.01 de março de 1991. Fixou-se o erro alfa (probabilidade de resultado "falso-positivo") em 5\% (intervalo de confiança de 95\%) e o erro beta (probabilidade de resultado "falso-negativo") em $20 \%$ (poder do estudo de $80 \%$ ). A razão dopplerfluxometria de ducto venoso normal/dopplerfluxometria de ducto venoso alterada era considerada 7:3. A freqüência esperada de fetos com gasometria anormal no grupo de dopplerfluxometria venosa normal foi de $20 \%$ e no grupo de dopplerfluxometria venosa comprometida foi de $90 \%$. Considerando estes parâmetros, o cálculo do tamanho amostral indicava a inclusão de 20 casos (14 normais e 6 alterados).

O estudo estatístico utilizado para analisar as variáveis categóricas (teste de significância da associação ou dependência) foi o $\chi^{2}$. O resultado foi considerado significativo para $\mathrm{p} \leq 0,05$.

Os pontos de corte da relação S/A da dopplerfluxometria do ducto venoso foram estabelecidos a cada variação de 0,2 desta relação. Foram calculadas: sensibilidade, especificidade, taxa de verdadeiro-positivos e taxa de falso-positivos para cada ponto de corte. Foi construída uma curva, chamada receiver operator characteristic (ROC) cur$v e$, plotando-se a taxa de verdadeiro-positivos (sensibilidade) contra a taxa de falso-positivos (1 - es- 
pecificidade) ao longo da faixa de pontos de corte. Os valores nos eixos vão de uma probabilidade de 0 a 1,0 (ou, alternativamente, de 0 a 100 por cento). O ponto de corte escolhido foi onde a curva ROC "muda de tendência".

\section{Resultados}

Foram selecionadas 60 gestantes no período de janeiro de 1998 a janeiro de 2003. A média das idades maternas foi de 28 anos $( \pm 1,9$ anos). No momento do estudo a idade gestacional variou entre 25 e 33 semanas, média de 29,7 semanas $( \pm 1,8$ semanas) e mediana de 30 semanas.

Todos os fetos apresentavam-se centralizados e, dentre eles, 14 apresentavam gasometria anormal ao nascimento e 46 apresentavam gasometria normal. A prevalência de resultados de fetos com gasometria anormal no material estudado foi de $23,3 \%$.

A média de peso dos conceptos foi de $913 \mathrm{~g}$ $( \pm 228 \mathrm{~g})$. A taxa de fetos PIG em nossa casuística foi de $81,1 \%$.

Observou-se associação significativa entre o ducto venoso anormal e gasometria anormal ao nascimento $\left(\chi^{2}=784,44, \mathrm{p}<0,00001\right)$. Pela avaliação da curva ROC, o ponto de corte escolhido (onde a curva "muda de tendência") correspondeu à relação S/A de 3,4 (Tabela 1 e Figura 1).

Tabela 1 - A primeira coluna corresponde aos valores da relação sístole ventricular/ sístole atrial (S/A) da dopplerfluxometria do ducto venoso, a cada intervalo de 0,2 , com seus respectivos cálculos de taxa de falso-positivos (1 -especificidade) e verdadeiropositivos (sensibilidade), na segunda e terceira coluna.

\begin{tabular}{ccc}
\hline S/A & 1 - Especificidade & Sensibilidade \\
\hline 1,60 & 91,40 & 100,00 \\
1,80 & 78,26 & 92,85 \\
2,00 & 56,52 & 78,57 \\
2,20 & 41,31 & 78,57 \\
2,40 & 32,61 & 78,57 \\
2,60 & 26,09 & 78,57 \\
2,80 & 21,74 & 78,57 \\
3,00 & 15,22 & 78,57 \\
3,20 & 13,05 & 78,57 \\
3,40 & 10,87 & 78,57 \\
3,60 & 10,87 & 78,57 \\
3,80 & 8,70 & 64,28 \\
4,00 & 8,70 & 50,00 \\
4,20 & 8,70 & 35,71 \\
4,40 & 6,53 & 35,71 \\
\hline
\end{tabular}

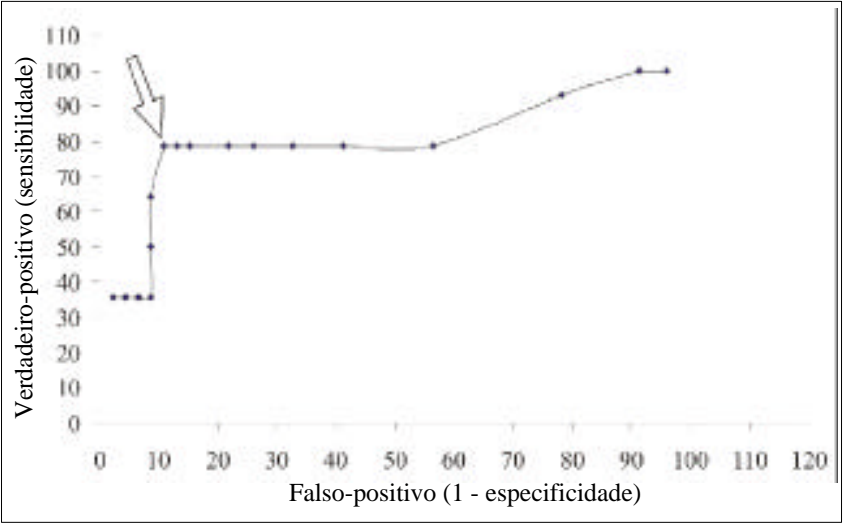

Figura 1 - Curva ROC (receiver operator characteristic). Plotou-se a taxa de verdadeiropositivos (sensibilidade) contra a taxa de falso-positivos (1 - especificidade) ao longo da faixa de pontos de corte, a cada intervalo de 0,2 da relação S/A da dopplerfluxometria do ducto enoso. A seta indica o ponto onde a cuva muda de tendência, correspondente ao valor da relação S/A de 3,4 .

A interpretação destes valores nos permite considerar que, em um feto centralizado, a dopplerfluxometria do ducto venoso (relação S/A maior do que 3 ,4) pode identificar de maneira não invasiva os conceptos que apresentarão gasometria anormal ao nascimento.

\section{Discussão}

A análise do território venoso pode ser útil se o desejado é aguardar a maturidade ou reduzir a morbidade quando estamos lidando com prematuridade extrema. A mortalidade neonatal global diminui com o aumento do peso ao nascimento ${ }^{21}$. Baschat et al. ${ }^{22}$ consideram que a idade gestacional é o parâmetro que apresenta maior impacto nos resultados pós-natais.

A dopplervelocimetria venosa alterada parece indicar deterioração fetal em meio intrauterino inóspito no qual o feto não parece ter condição de sobreviver por muito tempo. Precede as alterações de outros parâmetros biofísicos, como por exemplo as alterações da cardiotocografia, podendo ser parâmetro a se considerar isoladamente na decisão da interrupção da gestação ${ }^{23}$.

Dois mecanismos parecem ser responsáveis pelo padrão anormal das ondas de velocidade venosas: uma é o aumento da pós-carga ventricular direita e outra a falência miocárdica ${ }^{8}$. Demonstrou-se que a hipoxemia crônica está relacionada ao aumento do fluxo miocárdico. A capacidade do feto de compensar a redução do fluxo placentário pela centralização está relacionada à adequada oxigenação miocárdica, uma vez que a pós-carga está aumentada. Neste tempo, embora exista aumento da resistência na circulação feto- 
placentária, a centralização se dá sem que haja alteração nas ondas de dopplerfluxometria venosas. As alterações progressivas na circulação venosa indicam falência no mecanismo compensatório e o desenvolvimento de insuficiência cardíaca por hipoxia miocárdica ${ }^{24}$.

As pesquisas nesta área se justificam pelo fato de não sabermos por quanto tempo se deve monitorar um feto centralizado até que ele apresente sinais de comprometimento grave e se vale a pena investir tanto dinheiro e tempo, em aparelhos médicos e pesquisa, se não formos capazes de atuar antes que o sofrimento fetal ocorra ${ }^{19}$. O risco de lesão fetal secundária à deficiência de oxigênio e nutrientes precisa ser comparado ao risco atribuído à prematuridade, que pode ser grave problema antes da $32^{\mathrm{a}}$ semana de gestação. Fetos pequenos para a idade gestacional parecem ser capazes de tolerar hipoxemia crônica sem seqüelas, permitindo que o parto se dê mais tardiamente por meio da redistribuição do fluxo (centralização), desde que a avaliação da dopplerfluxometria venosa seja normal ${ }^{8}$.

Baschat et al. ${ }^{22}$ demonstraram, por análise multivariada, que só a idade gestacional apresenta correlação com complicações pós-natais, ao passo que a dopplerfluxometria venosa anormal só estava associada à morte intra-útero ${ }^{22}$. Entretanto, Ozcan et al. ${ }^{25}$ observaram que a dopplervelocimetria anormal do ducto venoso foi o único parâmetro que apresentou associação com mortalidade perinatal e índice de Apgar inferior a 5.

Com o propósito de identificar um ponto de corte a partir do qual poderemos identificar os conceptos comprometidos, desenvolvemos este trabalho. Todos os estudos disponiveis na literatura utilizam como critério de corte os valores superiores ao percentil 95 para a idade gestacional. A curva ROC mostra a severidade do contrabalanço entre a sensibilidade e a especificidade de um teste diagnóstico e é usada para auxiliar a decidir onde se localiza o melhor ponto de corte. Em nosso estudo o ponto de corte estabelecido corresponde ao valor da relação S/A do ducto venoso de 3,4.

Concordamos com Hecher et al. ${ }^{7}$ ao afirmarem que o exame dopplerfluxométrico do território venoso fetal pode ajudar na decisão do momento ideal para a interrupção da gestação em fetos centralizados. Entretanto, a idade gestacional desempenha papel importante na decisão médica, pois o risco de acidemia fetal grave precisa ser comparado ao risco da prematuridade extrema, ambas causas potenciais de mortalidade perinatal e morbidade pós-natal.

Este estudo nos permite concluir que a análise da relação S/A do ducto venoso se mostra ade- quada para a predição de gasometria anormal em fetos prematuros, centralizados, quando os valores são superiores a 3,4.

\section{ABSTRACT}

Objective: to assess through Dopllerfluxometry the S/A ratio of the ductus venosus and determine the cut-off point to identify preterm fetuses with the "brain sparing phenomenon".

Methods: a cross-sectional study was performed in 60 pregnant women that presented the "brain sparing phenomenon" (umbilical cerebral ratio $>1$ ) and gestational age between 25 and 33 weeks. The following parameters were studied: $S / A$ ratio of the ductus venosus, $p H$ and base excess (BE) of a fetal blood sample collected from the umbilical vein immediately after birth. The fetuses were classified according to the gas analysis result. They were considered abnormal when $\mathrm{pH}<7.20$ and $\mathrm{BE}<-6 \mathrm{mmol} / \mathrm{l}$. A receiver operator characteristic (ROC) curve analysis was performed to examine the relationship between $S / A$ ratio and fetal acidemia.

Results: sixty pregnant women in the period of January 1998 to January 2003 were selected. In the moment of the study the gestational age varied from 25 to 33 weeks, with an average of 29.7 weeks ( \pm 1.8 weeks). All of the fetuses presented the "brain sparing phenomenon". Among them 14 presented abnormal gas analysis at birth and 46 presented normal gas analysis. The prevalence of fetuses with abnormal gas analysis in the studied material was $23.33 \%$. Significant association was observed between the abnormal ductus venosus velocimetry and abnormal gas analysis at birth $\left(\chi^{2}\right.$ $=784.44, p<0.00001)$ in preterm fetuses with "brain sparing phenomenon". The best cut-off point of the S/A ratio (where the ROC curves bent) was 3.4.

Conclusion: fetal acidemia in preterm fetuses with "brain sparing phenomenon" may be noninvasively identified by Doppler measurement of the ductus venosus when the S/A rises above 3.4.

KEYWORDS: Ultrasonography, Doppler. Blood gas analysis. Placental circulation. Dopplerfluxometry.

\section{Referências}

1. Montenegro CAB, Rezende Filho J. Gestação de altorisco. In: Rezende J, editor. Obstetrícia. $8^{a}$ ed. Rio de Janeiro: Guanabara Koogan; 1998. p. 932-51.

2. Fanaroff AA, Wright LL, Stevenson DK, et al. Verylow-birth-weight outcomes of the National Institute of Child Health and Human Development Neonatal Research Network, May 1991 through December 1992. Am J Obstet Gynecol 1995; 173:1423-31. 
3. Cunningham FG, Gant NF, Leveno KJ, et al. Preterm birth. In: Williams Obstetrics. $21^{\text {st }}$ ed. New York: McGraw-Hill; 2001. p. 689-727.

4. Baschat AA, Gembruch U. The cerebroplacental Doppler ratio revisited. Ultrasound Obstet Gynecol 2003; 21:124-7.

5. Warshaw JB. Intrauterine growth retardation: adaptation or pathology? Pediatrics 1985; 76:998-9.

6. Hecher K, Snijders R, Campbell S, Nicolaides K. Fetal venous, intracardiac, and arterial blood flow measurements in intrauterine growth retardation: relationship with fetal blood gases. Am J Obstet Gynecol 1995; 173:10-5.

7. Hecher K, Campbell S, Snijders R, Nicolaides K. Reference ranges for fetal venous and atrioventricular blood flow parameters. Ultrasound Obstet Gynecol 1994; 4:381-90.

8. Hecher K, Campbell S. Characteristics of fetal venous blood flow under normal circumstances and during fetal disease. Ultrasound Obstet Gynecol 1996; 7:68-83.

9. Sherer DM, Fromberg RA, Divon MY. Prenatal ultrasonographic assessment of the ductus venosus: a review. Obstet Gynecol 1996; 88:626-32.

10.Sá RAM, Chaves Netto H, Amim Junior I, et al. Ductus venosus velocimetry in normal pregnancy. Int J Gynaecol Obstet 2000; 70 (Suppl 1): A28.

11.De Vore GR, Horenstein J. Ductus venosus index: a method for evaluating right ventricular preload in the second-trimester fetus. Ultrasound Obstet Gynecol 1993; 3:338-42.

12.Arduini D, Rizzo G. Normal values of pulsatility index from fetal vessels: a cross- sectional study on 1556 healthy fetuses. J Perinat Med 1990; 18:16572.

13.Rizzo G, Arduini D, Romanini C, Mancuso S. Doppler echocardiographic assessment of time to peak velocity in the aorta and pulmonary artery of small for gestational age fetuses. Br J Obstet Gynaecol 1990; 97:603-7.

14.Weiner Z, Farmakides G, Schulman H, Penny B. Central and peripheral hemodynamic changes of fetuses with absent end-diastolic velocity in umbilical artery: correlation with computerized fetal heart rate pattern. Am J Obstet Gynecol 1994; 170:509-15.
15.Rizzo G, Capponi A, Arduini D, Romanini C. Ductus venosus velocity waveforms in appropriate and small for gestational age fetuses. Early Hum Dev 1994; 39:15-26.

16.Sá RAM, Chaves Netto H, Lopes LM, Barreto MJV, Cabral ACV. Dopplerfluxometria de ducto venoso: relação com a gasometria em fetos prematuros com centralização de fluxo sangüineo. Rev Bras Ginecol Obstet 2003; 25:261-8.

17. Orlandi OV, Sabrá A. O recém-nascido prematuro. In: Rezende J, editor. Obstetrícia. 9 ${ }^{a}$ ed. Rio de Janeiro: Guanabara Koogan; 2002. p.1368-77.

18.Pennati G, Bellotti M, Ferrazzi E, Rigano S, Garberi A. Hemodynamic changes across the human ductus venosus: a comparison between clinical findings and mathematical calculations. Ultrasound Obstet Gynecol 1997; 9:383-91.

19.Surerus E, Pettersen H, Penido MT, Faria M. Nos caminhos da circulação fetal: o ducto venoso. Femina 2002; 30:433-8.

20.Vintzileos AM, Egan JFX, Campbell WA, et al. Asphyxia at birth as determined by cord blood $\mathrm{pH}$ measurements in preterm and term gestations: correlation with neonatal outcome. J Matern Fetal Med 1992; 1:7-13.

21.Lopes LM. Mortalidade neonatal na idade gestacional extremamente baixa (23 a 28 semanas) [tese]. Rio de Janeiro: Univ. Federal do Rio de Janeiro; 1997.

22.Baschat AA, Gembruch U, Reiss I, Gortner L, Weiner CP, Harman CR. Relationship between arterial and venous Doppler and perinatal outcome in fetal growth restriction. Ultrasound Obstet Gynecol 2000; 16:407-13.

23.Rizzo G, Capponi A, Arduini D, Romanini C. The value of fetal arterial, cardiac and venous flow in predicting $\mathrm{pH}$ and blood gases measured in umbilical blood at cordocentesis in growth retarded fetuses. Br J Obstet Gynaecol 1995; 102:963-9.

24.Kirkpatrick SE, Pitlick PT, Naliboff J, Friedman WF. Frank-Starling relationship as an important determinant of fetal cardiac output. Am J Physiol 1976; 231:495-500.

25.Ozcan T, Sbracia M, D'Ancona LR, Copel JA, Mari G. Arterial and venous Doppler velocimetry in the severely growth-restricted fetus and associations with adverse perinatal outcome. Ultrasound Obstet Gynecol 1998; 12:39-44. 